\title{
Long noncoding RNA H19 accelerates tenogenic differentiation by modulating miR-140- 5p/VEGFA signaling
}

\author{
You-Jie Liu, ${ }^{1}$ Hua-Jun Wang, ${ }^{1}$ Zhao-Wen Xue, ${ }^{1}$ Lek-Hang Cheang, ${ }^{2}$ Man-Seng Tam, ${ }^{3}$ Ri-Wang Li, ${ }^{1}$ Jie-Ruo Li, ${ }^{1}$ \\ Hui-Ge Hou, ${ }^{1}$ Xiao-Fei Zheng ${ }^{1}$ \\ ${ }^{1}$ Department of Orthopedic Surgery and Sports Medicine Center, The First Affiliated Hospital and The First Clinical \\ College, Jinan University, Guangzhou \\ ${ }^{2}$ Macau Medical Science \& Technology Research Association, Macau \\ ${ }^{3}$ IAN WO Medical Center, Macau Special Administrative Region, Macau, China
}

\begin{abstract}
Rotator cuff tear (RCT) is a common tendon injury, but the mechanisms of tendon healing remain incompletely understood. Elucidating the molecular mechanisms of tenogenic differentiation is essential to develop novel therapeutic strategies in clinical treatment of RCT. The long non-coding RNA H19 plays a regulatory role in tenogenic differentiation and tendon healing, but its detailed mechanism of action remains unknown. To elucidate the role of H19 in tenogenic differentiation and tendon healing, tendon-derived stem cells were harvested from the Achilles tendons of Sprague Dawley rats and a rat model of cuff tear was established for the exploration of the function of H19 in promoting tenogenic differentiation. The results showed that H19 overexpression promoted, while H19 silencing suppressed, tenogenic differentiation of tendon-derived stem cells (TDSCs). Furthermore, bioinformatic analyses and a luciferase reporter gene assay showed that H19 directly targeted and inhibited miR-140-5p to promote tenogenic differentiation. Further, inhibiting miR-140-5p directly increased VEGFA expression, revealing a novel regulatory axis between H19, miR-140-5p, and VEGFA in modulating tenogenic differentiation. In rats with RTC, implantation of H19-overexpressing TDSCs at the lesion promoted tendon healing and functional recovery. In general, the data suggest that H19 promotes tenogenic differentiation and tendon-bone healing by targeting miR-140-5p and increasing VEGFA levels. Modulation of the H19/miR-140-5p/VEGFA axis in TDSCs is a new potential strategy for clinical treatment of tendon injury.
\end{abstract}

Key words: IncRNA; miRNA; tendon stem cell; rotator cuff tear repair

Correspondence: Xiao-Fei Zheng, Department of Orthopedic Surgery and Sports Medicine Center, The First Affiliated Hospital, and The First Clinical College, Jinan University, Guangzhou, China.

E-mail: zhengxiaofei12@163.com

Contributions: HJW, XFZ, study design; YJL, ZWX, study performing; JRL, HGH, contribution to data analysis; LHC, MST, supervision of the experiment. All authors proved the final study. All authors read and approved the final manuscript.

Ethical Approval: All animal experiments were approved by the Ethics Committee of the First Affiliated Hospital, Jinan University, and were conducted in accordance with institutional guidelines for animal care and use.

Funding: This work was supported by the Project of Administration of Traditional Chinese Medicine of Guangdong Province of China (grant No. 20202040), the Fundamental Research Funds for the Central Universities (grant No. 21620452) and the National Natural Science Foundation of China (Grant No. 81973660).

Availability of data and materials: The datasets used and/or analyzed during the current study are available from the corresponding author on reasonable request.

Conflict of interest: The authors declare no conflict of interest. 


\section{Introduction}

Rotator cuff tear (RCT) is a common injury that causes shoulder joint pain and muscle weakness. ${ }^{1}$ Epidemiological studies have reported that $20 \%$ of people over 20 years of age and up to $25 \%$ of people over 50 years of age suffer from rotator cuff injury. ${ }^{2,3}$ Rotator cuff injury is a primary cause of shoulder joint pain and dysfunction, and severely limits work efficiency and quality of life. ${ }^{4}$ In Italy, 62.1 out of every 100,000 people have undergone rotator cuff repair surgery, and according to prediction models, the total amount spent on RCT in 2025 will reach one billion euros. ${ }^{5}$ Previously, surgical treatment of RCT was thought to have satisfactory efficacy and outcomes, but recent studies have identified that the long-term efficacy of surgical treatment is uncertain, ${ }^{6}$ and that conservative (non-surgical) treatment in fact reduced pain and improved function in patients with mild to moderate $\mathrm{RCT}^{7}$ About $11-36 \%$ of patients with RCT suffered from re-rupture due to unhealed tendon-bone injuries. ${ }^{6}$ Thus, elucidating the detailed mechanisms of tendon injury and recovery to develop novel approaches to RCT treatment is a significant unmet clinical need.

Tendon-derived stem cells (TDSCs) were first identified in rat and human tendons in 2007. ${ }^{89}$ As tenocyte precursor cells, TDSCs exhibit similar biological functions to mesenchymal stem cells (MSCs). ${ }^{10}$ TDSCs exhibit strong multi-directional differentiation potential into tendon, fat, osteogenic, and cartilage tissues. ${ }^{11}$ Promoting differentiation of TDSCs into tendon cells and inhibiting differentiation of TDSCs into non-tendon cells under pathological conditions have been identified as effective strategies for treatment of RCT. ${ }^{12}$

miRNAs are short non-coding RNA molecules 17-25 nucleotides in length, and control gene product levels by suppressing translation of target mRNAs, and in some cases by decreasing stability of target mRNAs. miRNAs are involved in a variety of physiological and pathological processes. An increasing body of evidence suggests that miRNAs are closely related to tendinopathy and have therapeutic potential in this context. For example, miRNA-499 is significantly upregulated in samples from injured tendons, and functions by targeting CELF2 and MYB. ${ }^{13}$ In another set of miRNA screening experiments for degenerative RCT, expression levels of miR-29a and miR-29c were significantly downregulated in RCT patient serum and biopsy samples. ${ }^{14}$ In human tendon stem/progenitor cells (TSPCs), miR-140-5p regulates Pin1 expression, which is decreased during TSPC aging. ${ }^{15}$ However, the roles and mechanisms of miR-140-5p in TDSC development and tendon tear repair remain largely unknown.

Long non-coding RNA (lncRNA) plays an important role in transcriptional and post-transcriptional regulation of gene expression, and is a regulatory hub for transcriptional activity and mRNA expression of target genes. Several studies have suggested a relationship between IncRNA and tendon disease, but the regulatory mechanisms remain incompletely understood. Zheng et al. identified that eight dysregulated lncRNAs could be involved in fiber formation after tendon injury. ${ }^{16}$ Inhibition of $\operatorname{lncRNA}$ KCNQ1OT1 suppressed the adipogenic and osteogenic differentiation of TDSCs via miR-138/PPARg/RUNX2 signaling, ${ }^{17}$ and IncRNA $\mathrm{H} 19$ has been reported to accelerate TDSC differentiation, thereby promoting tendon development and repairing tendon injuries through the TGF pathway. ${ }^{18}$ Growth factors such as VEGFA are major mediators of tendon-bone healing. They promote collagen formation, angiogenesis, and extracellular matrix formation, which are critical for tissue healing and regeneration. ${ }^{19}$ VEGFA belongs the VEGF protein family (VEGF-A, VEGF-B, VEGF-C, VEGF-D, and placental growth factor, PGF), and has great potential in tendon-bone healing.

In the present study, we used in vitro cultured TDSCs and an in vivo RCT rat model to examine the regulatory relationship between H19 and miR-140-5p in tenogenic differentiation during tendon injury recovery.

\section{Materials and Methods}

\section{Animals}

SD rats were supplied by the Institute of Laboratory Animal Science of Jinan University.

\section{TDSC isolation and cell culture}

TDSCs were harvested from the Achilles tendons of SD rats as previously described. ${ }^{20}$ Briefly, rat Achilles tendons were digested with type I collagenase (Sigma-Aldrich, St. Louis, MO, USA) and incubated for $2 \mathrm{~h}$ at $37^{\circ} \mathrm{C}$. Dissociated cells were plated in $100 \mathrm{~mm}$ dishes at 200 cells $/ \mathrm{cm}^{2}$, and cultured in DMEM (Gibco, Thermo Scientific, Waltham, MA, USA) with 20\% FBS (Gibco), 100 U/mL penicillin (Gibco) and $100 \mathrm{U} / \mathrm{ml}$ streptomycin (Gibco) for 10 days in a standard tissue culture incubator. TDSCs at passage 3 or 4 were used for subsequent experiments. TDSCs were confirmed as previously reported. ${ }^{20}$

\section{Rat RCT model}

Briefly, a longitudinal incision of approximately $1.5 \mathrm{~cm}$ was made on the anterolateral side of the shoulder. The supraspinatus tendon and muscle were exposed, and then half of the tendons were cut and sharply separated from the greater tuberosity. Subsequently, the supraspinatus tendons were sutured layer by layer using a 4-0 suture. Rats were randomly assigned into control, TDSC, or H19overexpressing TDSC groups, with 10 rats in each group. In the control group, rats were injected with $200 \mu \mathrm{L}$ fibrin glue in the tendon-bone interface. In the TDSC groups, fibrin glue with $2 \times 10^{6}$ TDSCs or H19-TDSCs was injected. Four or eight weeks later, tendons were subjected to biomechanical testing.

\section{Biomechanical testing}

Four or eight weeks after RCT, complete tendons of the rat supraspinatus muscle and proximal humerus were collected and subjected to biomechanical testing using an MTS 858 material testing system (MTS System, Minneapolis, MN, USA). A 0-5 N preload was applied. Samples were loaded with a crosshead speed of $14 \mathrm{~mm} / \mathrm{s}$. Ultimate force at failure $(\mathrm{N})$ and stiffness $(\mathrm{N} / \mathrm{mm})$ were then measured and recorded using a Sigma-Aldrich Plot 8.0 (SigmaAldrich). Ultimate force was determined from the load-displacement curve. Stiffness was calculated by measuring the slope.

\section{Generation of stable TDSCs}

H19 overexpression plasmid and shRNA sequences were cloned into the lentiviral vector $\mathrm{pLKO}$.1. Viruses were then transfected into HEK293 cells for packaging. Supernatants containing retrovirus particles were then collected and filtered using $0.45 \mu \mathrm{m}$ Millex-HV filters (Millipore, Bedford, MA, USA) to remove cell debris. To construct stable cell lines, TDSCs were infected with vector control lentivirus, H19 overexpressing lentivirus, shRNA control lentivirus, or shH19 lentivirus; $48 \mathrm{~h}$ after infection, cells were selected by 2 $\mu \mathrm{g} / \mathrm{mL}$ puromycin (Sigma-Aldrich), and the efficiency was determined by real-time quantitative PCR (RT-qPCR) assay. shRNA targeting sequences were as follows: shH19 5'GTGCAGGTAGAGCGAGGTAAA- ${ }^{\prime} \cdot{ }^{21}$

\section{Cell transfection}

miR-140-5p mimic (5'-ACCAUAGGGUAAAACCACUGUU- 
3'), miR-140-5p inhibitor (5'-CUACCAUAGGGU AAAACCACU-3'), and mimic and inhibitor negative controls (NCs) (5'-UUCUCCGAACGUGU CACGUTT-3') were purchased from GenePharma (Shanghai, China) and transfected with Lipofectamine 2000 (Thermo Fisher Scientific) according to the manufacturer's protocol.

\section{Sirius red staining}

A Sirius red staining assay was applied to measure collagen formation. Briefly, TDSCs were cultured in a 12-well plate. For cell harvesting, medium was removed, cells were washed and fixed with $4 \%$ paraformaldehyde for $40 \mathrm{~min}$, and subsequently incubated with $0.1 \%$ Sirius Red F3BA in a saturated picric acid solution for $45 \mathrm{~min}$. The plate was measured by using a spectrophotometer (BioTek Instruments, Winooski, VT, USA) at $540 \mathrm{~nm}$ to quantify the red color.

\section{Luciferase reporter assay}

To generate luciferase reporter constructs for H19 and VEGFA, the pGL3 vector (Promega, Madison, WA, USA) was used. H19 or VEGFA (wild-type, WT or mutant, MUT) regions against the binding site of miR-140-5p were cloned into the pGL3 vector. Binding site mutants were generated using a Site-Directed Mutagenesis kit (Thermo Fisher Scientific). Plasmids with an internal reporter (Renilla) were then co-transfected into 293T cells using the Dual Luciferase Assay System (Promega) according to the manufacturer's instructions. Fragments of miR-140-5p mimic, miR-140-5p inhibitor, or NC were co-transfected; 48 h later, cells were harvested, and luciferase activities were tested using a Luciferase Assay System (Promega) and normalized to the internal control signal.

\section{RNA isolation and RT-qPCR}

Total RNA was extracted using TRIzol reagent (Invitrogen, Carlsbad, CA, USA) according to the manufacturer's instructions. To reverse transcribe mRNA, $1 \mu \mathrm{g}$ RNA was used to reverse-transcribe mRNA into cDNA with an EasyScript cDNA Synthesis SuperMix (TransGen Biotech, Beijing, China) was used. For miRNA cDNA synthesis, a TransScript miRNA First-Strand cDNA Synthesis SuperMix (TransGen) was used. RT-qPCR was performed using TransStart Top Green quantitative PCR SuperMix (TransGen). Primer sequences used are listed in Table 1. For quantification, genes were normalized to Gapdh and miRNA to U6.

\section{Western blotting}

Samples were lysed using RIPA buffer with protease inhibitors (Beyotime, China). Proteins were then separated by SDS-PAGE $(10 \%)$ and transferred to PVDF membranes (Millipore). Membranes were blocked with milk and incubated with primary antibodies against VEGFA (\#ab46154; Abcam, Cambridge, UK) and GAPDH (\#ab8245, Abcam). After secondary antibody (Abclonal Biotechnology, Wuhan, China) incubation for $1 \mathrm{~h}$ at room temperature, blots were detected using enhanced chemiluminescence (ECL) (Beyotime). GAPDH was used as a loading control.

\section{Statistical analyses}

Statistical analyses were performed using SPSS (27.0). All data are presented as mean $\pm \mathrm{SD}$. A two-tailed Student's $t$-test (for two groups) or analysis of variance test (ANOVA, for multiple groups, One-Way ANOVA with Tukey post-hoc test, or Two-Way ANOVA with Bonferroni post-hoc test) was used to compare unpaired samples; $\mathrm{p}<0.05$ was considered statistically significant.

\section{Results}

\section{Involvement of $\mathrm{H19}$ in tenogenic differentiation}

TDSCs have reparative properties in tendon injury, exhibiting spontaneous tenogenic differentiation potentials in vitro. ${ }^{22} \mathrm{H} 19$ has previously been reported to promote tenogenic differentiation of human TDSCs. ${ }^{18}$ To confirm this, rat TDSCs were isolated and cultured, and stable H19-overexpression (H19) and H19-shRNA (shH19) TDSCs were generated using a lentiviral system and confirmed by RT-qPCR. TDSCs cells were treated with TGF- $\beta$ to induce tenogenic differentiation as previously reported. ${ }^{23,24} \mathrm{As}$ shown in Figure 1A, RT-qPCR demonstrated that the expression of H19 was markedly increased. In the H19 overexpression group, collagen formation significantly increased, as revealed by Sirius red staining (Figure 1B and Supplementary Figure 1A for enlarged details). mRNA levels of tenogenic markers and extracellular matrix (ECM) markers, including Scx, Mkx, Cola1, Fmod, Tnmd, and Dcn, were significantly upregulated in the H19 overexpression group (Figure 1C). Contrastingly, H19 expression was decreased in the shH19 group (Figure 1D and Supplementary Figure 1B for enlarged details). Genetic silencing of $\mathrm{H} 19$ resulted in decreased collagen formation (Figure 1E), and decreased levels of tenogenic and ECM

Table 1. Primer sequences for real-time qPCR.

\begin{tabular}{lll} 
Gene & Forward $\left(5^{\prime}-3^{\prime}\right)$ & Reverse $\left(5^{\prime}-3^{\prime}\right)$ \\
H19 & TAAAGCAGCTGGGGTGGTGAG & TGACTGGCAGGCACATCCAC \\
\hline Mkx & AGCCCAAACAGATCTGCACCTT & CTTCCACCTTCACTAGTGGCATCA \\
Tnmd & CTCAAGGACAACCTCAGCCTGAG & CGTTGCCTGAACATACTTGTTGAC \\
Collal & ATGGGTGGTCCCACAAGTGAA & CTCTCATCCAGCATGGGATCAA \\
Col $2 a l$ & GTCCGAGGTCCTAATGGAGATGC & GGTCCAGGGATCCGATGT \\
\hline Fmod & CCAGGTCCTGCTGGAAAA & CCTCTTTCTCCGGCCTTT \\
Den & CAAGGCAACAGGATCAATGAG & CTGCAGCTTGGAGAAGTCA \\
Vegfa & GACTCCACGACAATGAGATCACC & GTTGCCATCCAGATGCAGTTC \\
\hline
\end{tabular}


markers (Figure 1F). These data suggest that H19 is closely related to tenogenic differentiation, which is consistent with previous reports.

\section{H19 targets miR-140-5p to promote tenogenic differen- tiation}

Next, we sought to determine the detailed mechanism of H19 regulation of tenogenic differentiation. Using bioinformatic tools Starbase-ENCORI, ${ }^{25}$ we screened candidate miRNAs and found a putative H19 binding site on miR-140-5p (Figure 2A). Overexpression of a miR-140-5 $p$ mimic significantly decreased, while a miR-140-5p inhibitor increased, expression of H19 in TDSCs (Fig. 2B). To further assess the potential direct interaction between H19 and miR-140-5p, a dual-luciferase reporter gene assay was used. miR-140-5p significantly reduced the luciferase activity of H19 WT but not mutant H19 (H19 MUT) (Figure 2C). Furthermore, miR-140-5p mimics were transfected into TDSCs with or without $\mathrm{H} 19$ overexpressing plasmids. Overexpression of miR$140-5 p$ significantly suppressed tenogenic differentiation with TGF- $\beta$ treatment, as demonstrated by Sirius red staining (Figure 2D and Supplementary Figure 1C for enlarged details). However, additional overexpression of H19 markedly abolished the suppressive effect of miR-140-5p (Figure 3D). Taken together, these findings demonstrate that miR-140-5p directly interacts with H19 during tenogenic differentiation.

\section{miR-140-5p targets VEGFA}

Next, we sought to determine the potential downstream target genes of miR-140-5p. We screened candidate targets by bioinformatics prediction via TargetScan $^{26}$ and $\mathrm{ENCORI}^{25}$ and identified VEGFA as a gene of interest, as its 3'-UTR region contained a miR-140-5p target sequence (Figure 3A). To assess the direct relationship between VEGFA and miR-140-5p, a dualluciferase reporter assay was performed. miR-140-5p mimic transfection significantly decreased the wild-type VEGFA (VEGFA WT) reporter but not the mutant VEGFA reporter (VEGFA MUT) (Figure 4B). Furthermore, mRNA (Figure 3C) and protein (Figure 3D) levels of VEGFA were measured in the presence and absence of miR-140-5p transfection. miR-140-5p mimics significantly suppressed, while miR-140-5p inhibitors promoted, expression of VEGFA (Figure 3 C,D). These data indicate that miR-140-5p directly targets VEGFA.

\section{H19/miR-140-5p regulates VEGFA expression to pro- mote tenogenic differentiation}

After determining the relationships between H19/miR-140-5p
A

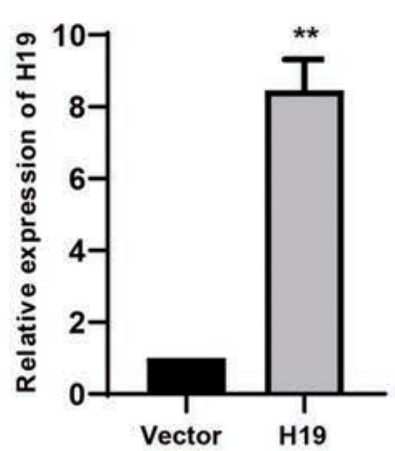

D

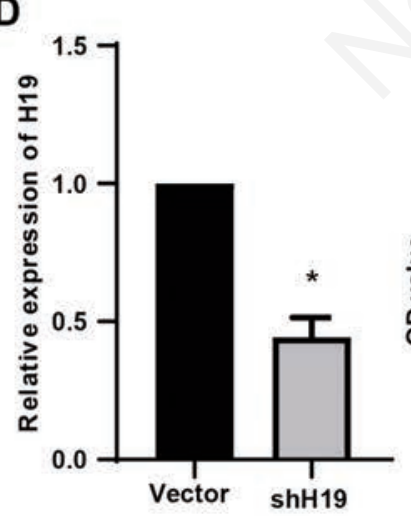

B
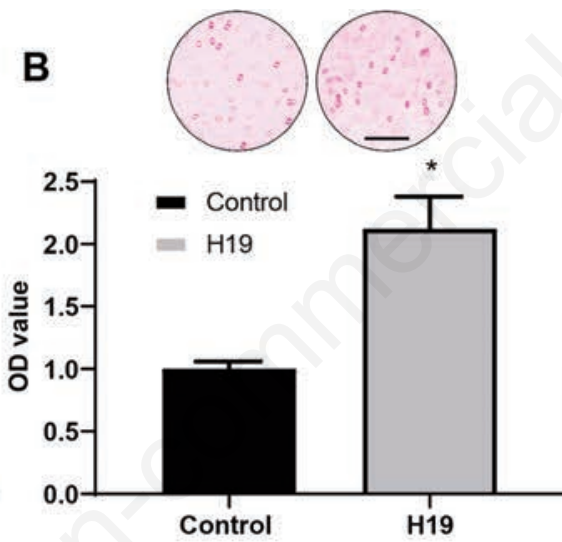

E

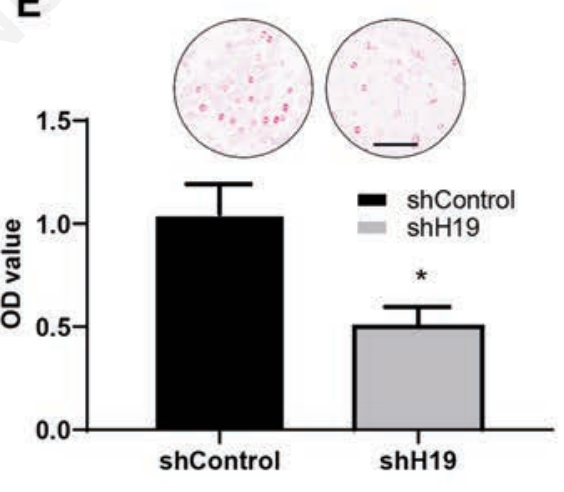

C

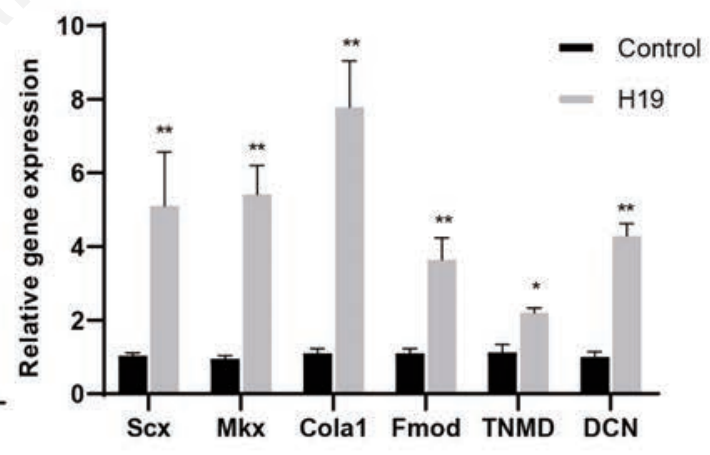

$\mathbf{F}$

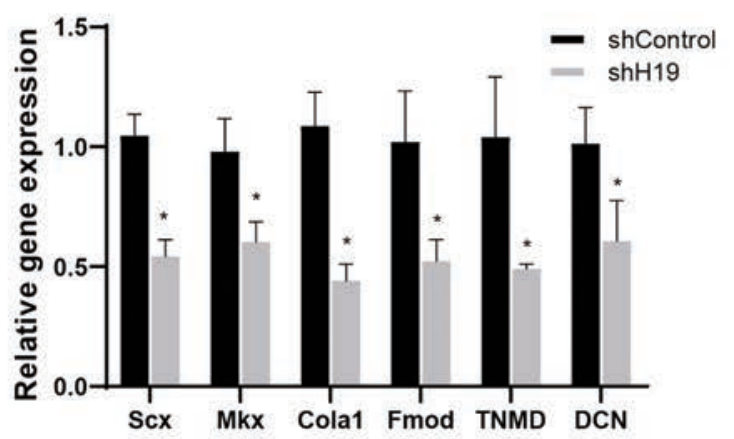

Figure 1. Figure 1. H19 regulates tenogenic differentiation of TDSCs. A) H19 was upregulated in TDSCs by H19-encoding plasmids. B) Sirius red staining of Control and H19-overexpressing TDSCs; scale bar: $20 \mu \mathrm{m}$. C) RT-qPCR detection of gene expression of tenogenic markers, including Scx, Mkx, Cola1, Fmod, Tnm, and Dcn, during tenogenic differentiation of TDSCs in the specified groups. D) H19 expression was decreased in TDSCs by plasmids containing H19 shRNA. E) Sirius red staining of shControl and shH19 groups; scale bar: $20 \mu \mathrm{m}$. F) mRNA expression level of tenogenic markers in shControl and shH19 groups; ${ }^{*} \mathrm{p}<0.05$; ${ }^{* *} \mathrm{p}<0.01$, relative to Vector or shControl group. 
and miR-140-5p/VEGFA, we determined if H19/miR-140-5p functioned via VEGFA to regulate tenogenic differentiation. A dualluciferase VEGFA reporter assay was conducted with or without miR-140-5p mimic or H19 overexpression plasmids (Figure 4A). miR-140-5p significantly reduced the level of VEGFA reporter, which was reversed by H19 overexpression (Figure 4A). Moreover, mRNA (Figure 4B) and protein (Figure 4C) levels of VEGFA were measured in these treatment groups. Both the mRNA and protein levels of VEGF were decreased by miR-140-5p mimic transfection, which was reversed by H19 co-transfection (Figure 4C). Furthermore, miR-140-5p significantly inhibited tenogenic differentiation, and this effect was rescued by H19 overexpression (Figure 4D and Supplementary Figure 1D for enlarged details). Taken together, these data indicate that the H19/miR-140-5p axis regulates VEGFA during tenogenic differentiation.

A
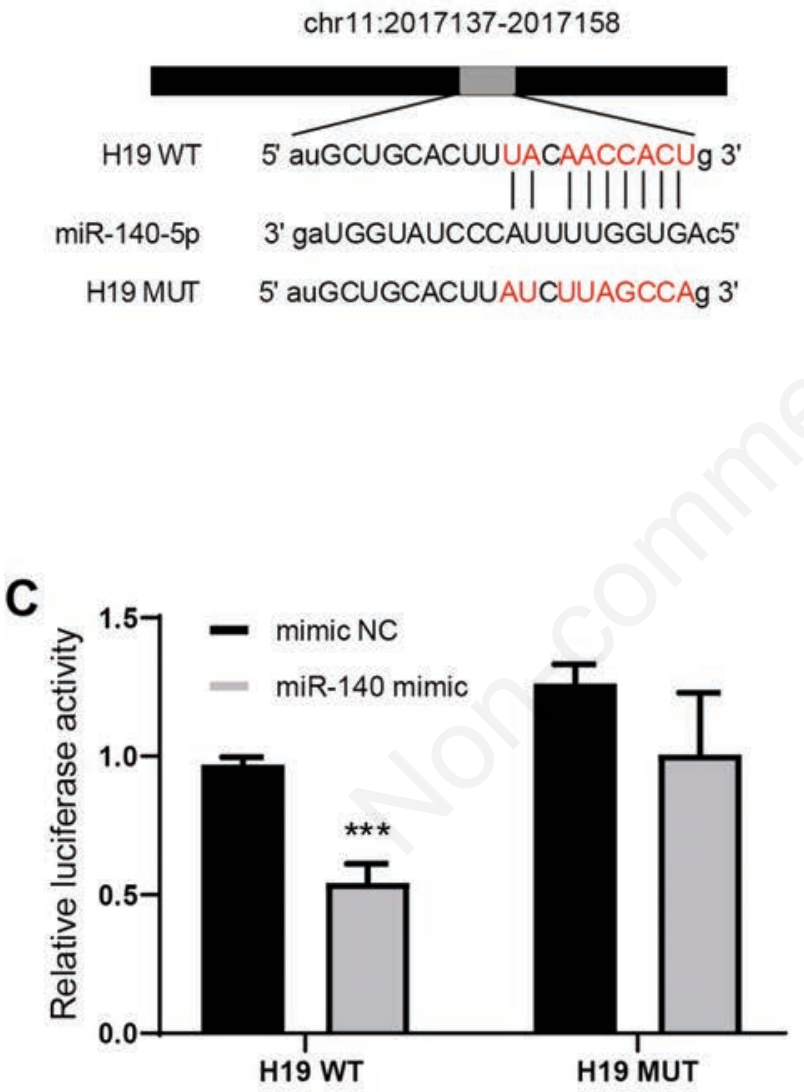

\section{H19 overexpression promotes rotator cuff tear repair}

To further evaluate the role of H19-promoted tenogenic differentiation, a rat RCT model was used. Rats with surgically induced RCT were injected with vehicle, normal TDSCs, or H19overexpressing TDSCs (H19-TDSCs) at the lesion site. Biomechanical testing was then performed 4 or 8 weeks later. Ultimate load to failure was significantly improved in the H19TDSC group relative to the normal TDSC group at both 4 and 8 weeks post-RCT (Figure 5A). However, stiffness was significantly increased by H19-TDSCs at week 8 but not week 4 post-RCT (Figure 5B). Tendon samples of the RCT site were collected and subjected to RT-qPCR. mRNA levels of CollA1 were significantly increased in both the TDSC and H19-TDSC groups relative to control on week 4 and week 8 , and were further increased in the H19-TDSC group relative to the TDSC group at both time points (Figure 5C). mRNA levels of Col2al followed the same trend (Figure 5D). These data indicate that $\mathrm{H} 19$ overexpression enhances

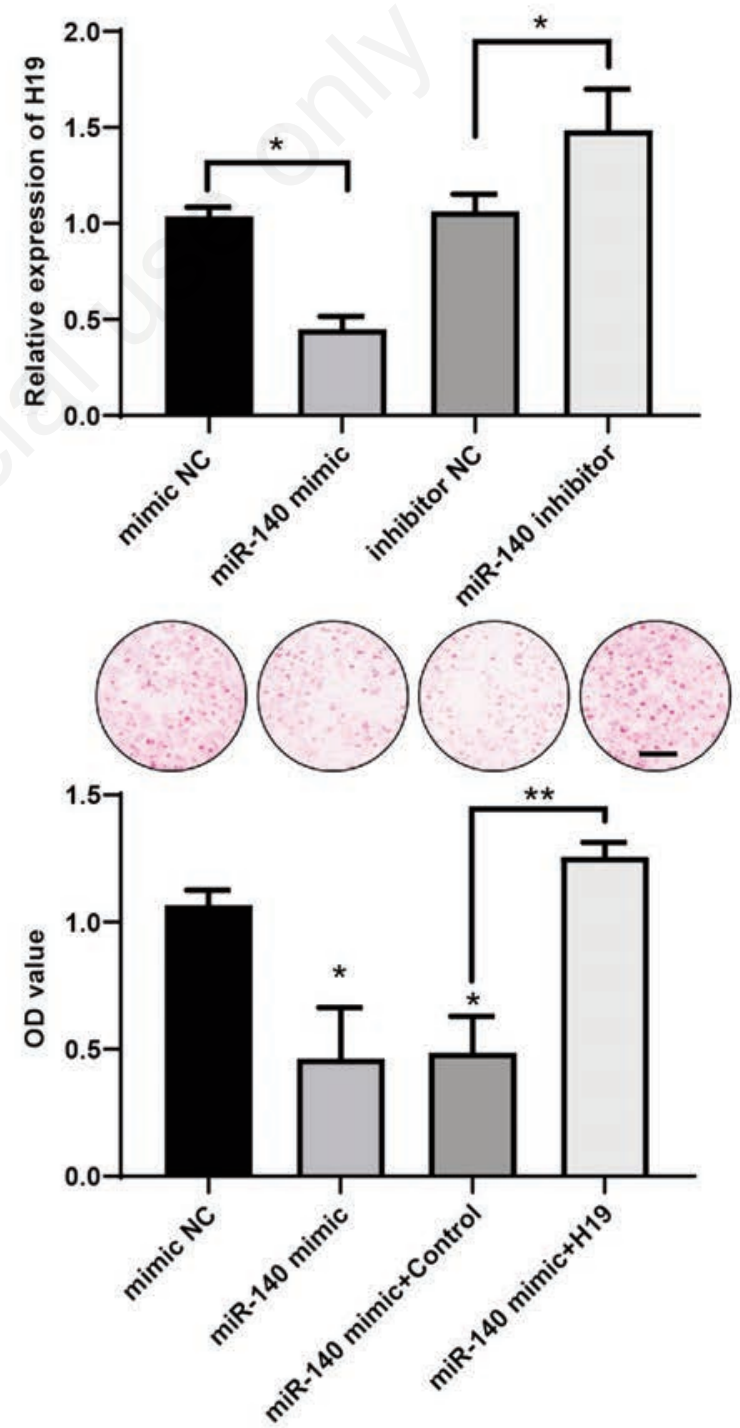

Figure 2. H19 directly targets miR-140-5p. A) Bioinformatic prediction of target sites in H19 against miR-140-5p. B) The RNA level of $\mathrm{H} 19$ in TDSCs was detected by RT-qPCR upon transfection of miR-140-5 mimic/inhibitor or negative control (NC). C) TDSCs were transfected with luciferase reporter plasmids containing H19 wild-type (WT) or mutant (MUT) gene, with or without miR-140$5 \mathrm{p}$ mimic or NC; luciferase activity was then detected. D) miR-140-5p mimic or NC were transfected into TDSCs; Sirius red staining was performed to assess tenogenic differentiation; scale bar: $20 \mu \mathrm{m} ;{ }^{*} \mathbf{p}<0.05 ;{ }^{* *} \mathrm{p}<0.01$. 
the protective effect of TDSCs during tendon-bone healing of rat RCT.

\section{Discussion}

In the present study, we demonstrated that H19 is involved in tenogenic differentiation. H19-overexpressing TDSCs promoted functional recovery of rat RCT. Bioinformatic analyses and luciferase reporter assays demonstrated that H19 targeted miR-140$5 \mathrm{p}$, and miR-140-5p directly targeted VEGFA. Furthermore, H19/miR-140-5p regulated VEGFA expression during tenogenic differentiation of TDSCs. Taken together, these results point to a novel role of the H19/miR-140-5p/VEGFA axis in tenogenic differentiation of TDSCs in RCT repair.

VEGF is highly upregulated at the surgical sites of leg bones in rats $^{27}$ and during the reconstruction of the cruciate ligament in dogs, Vegf mRNA levels peak at about 10 days after surgery. ${ }^{28}$ VEGF and BEGF are highly expressed in the bone-tendon junction during acute injury healing. ${ }^{19}$ Furthermore, VEGF-overexpressing MSCs promote tendon-bone healing of RCT by modulating miR-205-5p. ${ }^{29}$ Engineered nanoparticles carrying the VEGFA gene significantly promote tendon-bone healing in flexor tendon repair models. ${ }^{30,31}$ Collectively, these findings demonstrate an important role for VEGFA in regulating tendon-bone healing. In the present study, we found that VEGFA was upregulated in RCT, and that modulating H19/miR-140-5p directly affected VEGFA expression in TDSCs. However, VEGFA is only one of many downstream growth
A

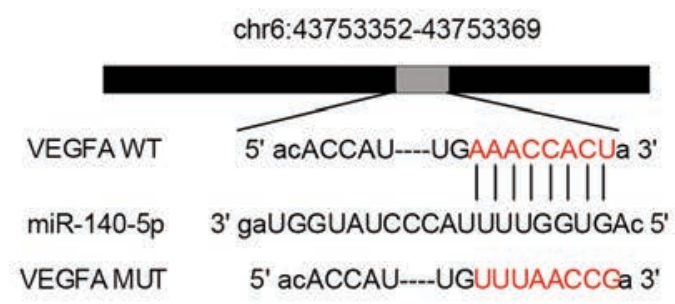

C

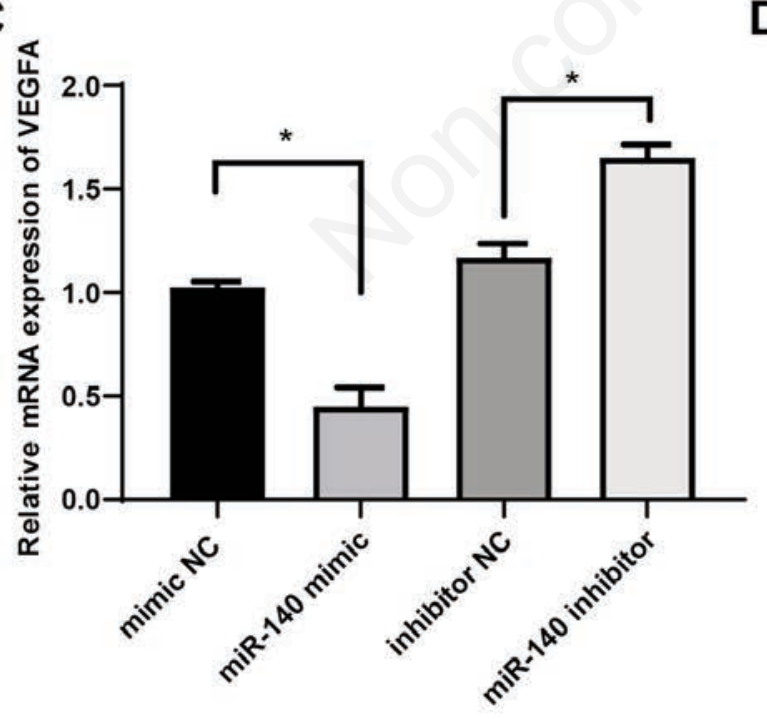

B
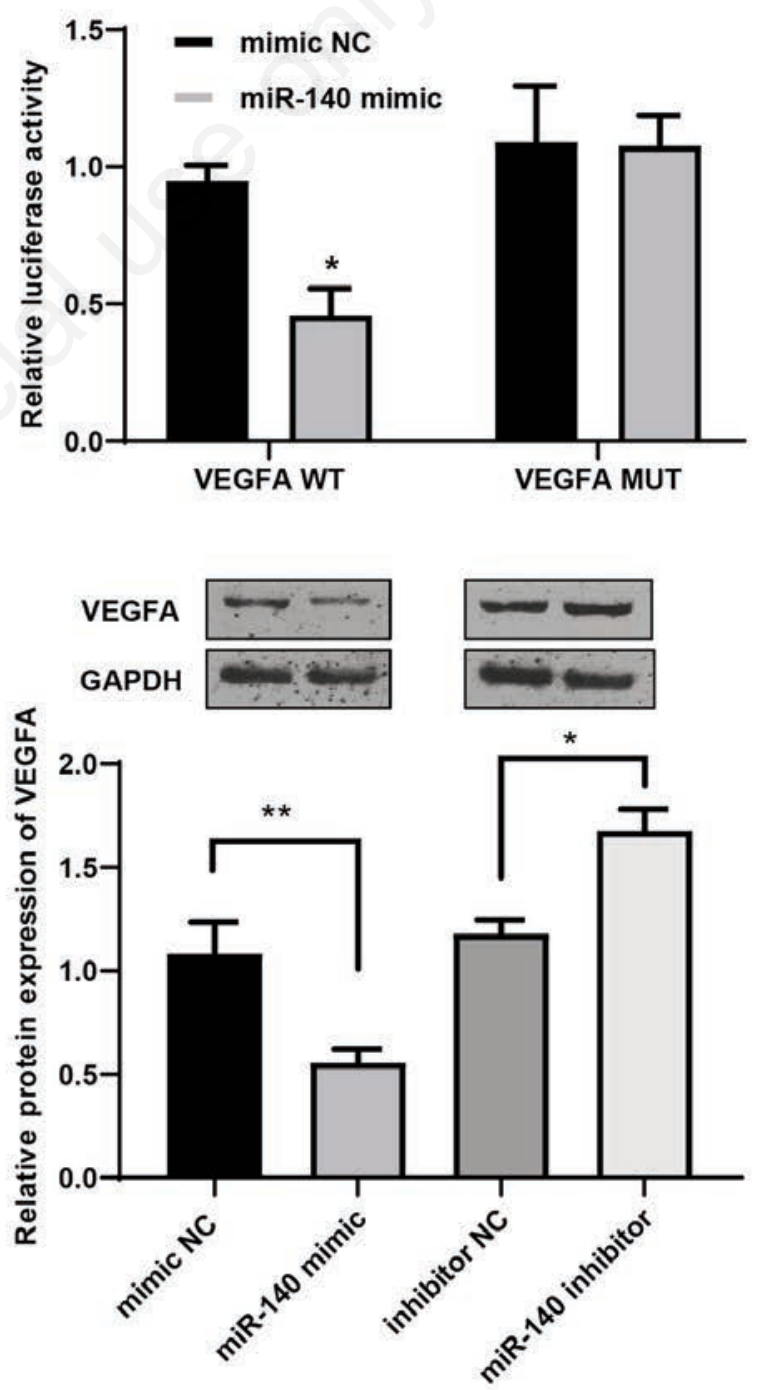

Figure 3. VEGFA is a direct target of miR-140-5p. A) Bioinformatics analysis of target sites for miR-140-5p in the VEGFA sequence. B) Luciferase analysis assay to assess the relationship between miR-140-5p and VEGFA. mRNA (C) and protein (D) levels of VEGFA were detected when miR-140-5p mimic/inhibitor or NC fragments were transfected into TDSCs. ${ }^{*} \mathrm{p}<0.05,{ }^{* *} \mathrm{p}<0.01$. relative to mimic/inhibitor NC group. 
effectors, and we postulate that the upstream switch of H19 may play an important role in regulating tenogenic differentiation and tendon-bone healing of RCT by regulating multiple downstream targets.

Traumatic injuries and degeneration of the rotator cuff are major causes of shoulder pain and dysfunction, causing chronic pain and restricted mobility. ${ }^{32}$ As the aging population increases, shoulder pain in the elderly is projected to become an increasing socioeconomic burden. Both traumatic and degenerative RCT are age-related, ${ }^{2}$ and are linked to intrinsic and extrinsic patient-specific factors. miRNAs have been identified as potential biomarkers and diagnostic tools for RCT. ${ }^{33}$ miRNAs regulate tenocyte function and tendon-related gene expression during tendon development and injury repair. ${ }^{34}$ For example, during the inflammatory response of RCT, miR-25 is downregulated in chronic rotator cuff tendinopathy. miR-25 plays a critical role during inflammatory responses via modulating the downstream target HMGB1, which is upregulated in RCT. ${ }^{35} \mathrm{NF}-\mathrm{kB}$ is also an important mediator of chronic and acute tendon injuries. ${ }^{36,37}$ miR-140-3p is significantly suppressed in both chronic tendinopathy and degenerative RCT, and miR-140-3p also negatively regulates NF-kB inflammatory signaling by inhibiting target gene expression of NCOA1 and NRIP1. ${ }^{38}$ Thus, miR-140 could promote inflammation during tendon injuries. In the present study, we demonstrated that miR-140-5p was downregulated in RCT samples and TDSCs, which was consistent with the former observation of miR-140-3p. Furthermore, suppressing miR-140-5p upregulated expression of the downstream target VEGFA to promote the tenogenic differentiation of TDSCs. The role of miR$140-5 p$ in regulating the inflammatory response in RTC will be investigated in future studies.

The functions of miRNAs in tendinopathy are a topic of intense investigation, but the role of lncRNAs remains largely obscure..$^{39,40}$ Mandy and colleagues used deep sequencing of transcriptome analysis to identify that several lncRNAs are changed in uninjured
A

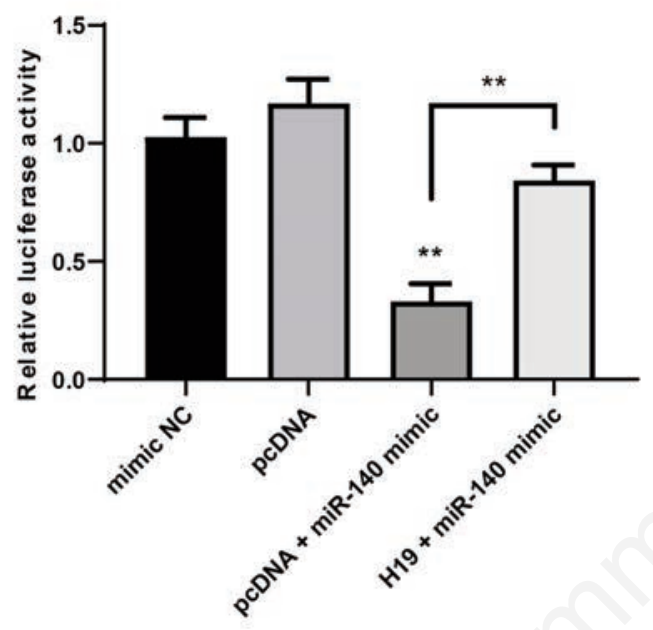

C

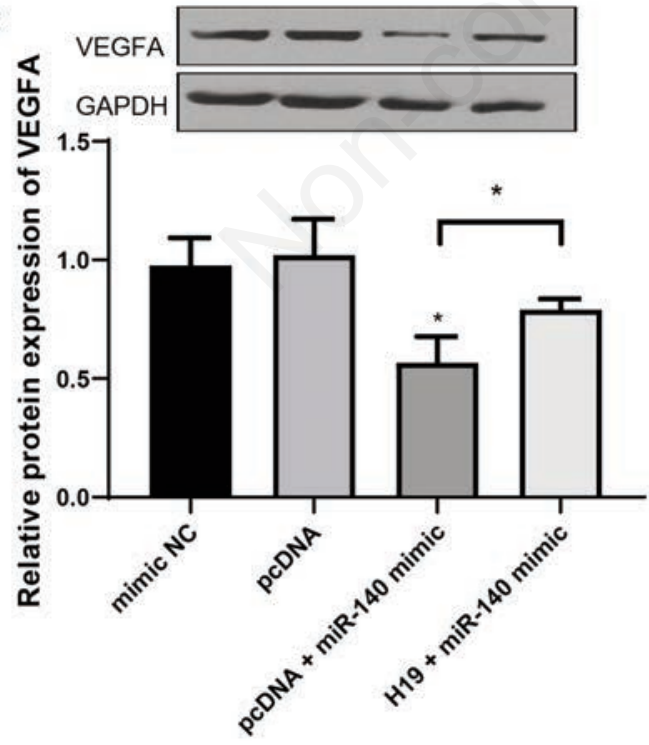

B

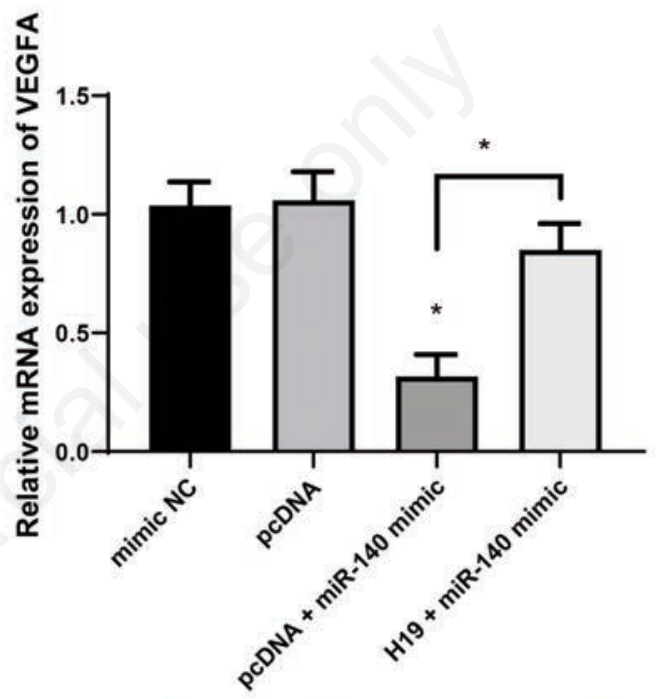

D

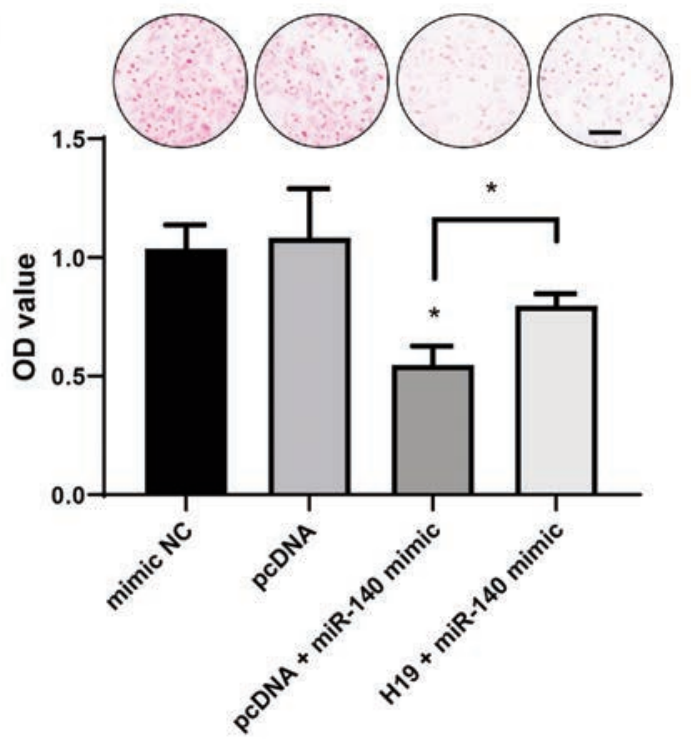

Figure 4. The H19/miR-140-5p/VEGFA axis regulates tenogenic differentiation. A) A VEGFA luciferase assay was performed when miR140-5p mimic or NC fragments were transfected with or without $\mathrm{H} 19$ overexpression plasmids. mRNA (B) and protein (C) levels of VEGFA with or without miR-140-5p mimics or H19 overexpression plasmids were determined by RT-qPCR and western blotting, respectively. D) Sirius red staining was performed to assess the tenogenic differentiation effect in TDSCs treated as in (A); scale bar: 20 $\mu \mathrm{m} .{ }^{*} \mathbf{p}<0.05,{ }^{* *} \mathbf{p}<\mathbf{0 . 0 1}$ relative to mimic $\mathrm{NC}$ or the indicated group. 


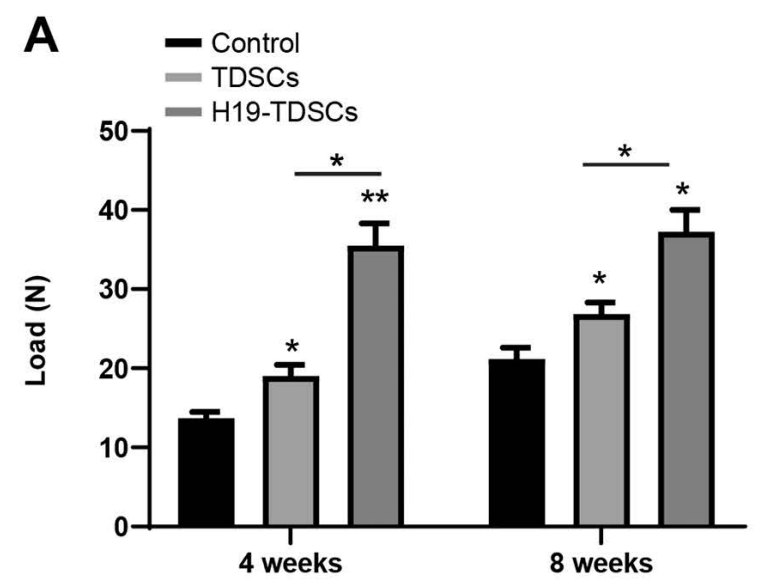

B
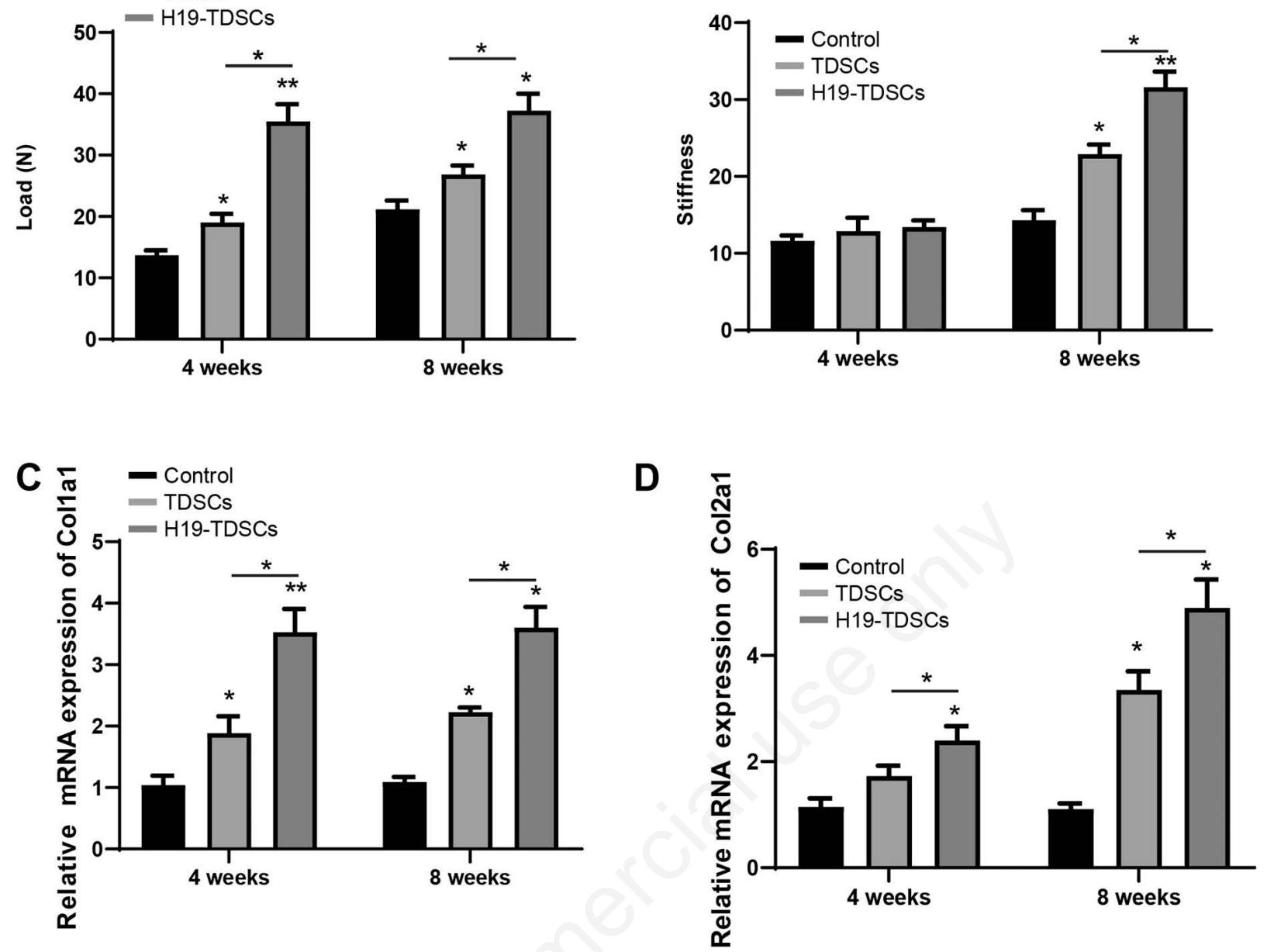

Figure 5. H19 overexpression increases the protective effect of TDSCs in tendon-bone healing of RCT. Lentiviral H19 overexpression vectors were introduced to TDSCs to generate a stable cell line. Cells were then injected into the lesions of RCT rats. Four and eight weeks later, tendons were subjected to mechanical testing. A) Ultimate load to failure in the H19-TDSC group was significantly increased relative to the control group at the 4- and 8-week points. B) Stiffness markedly increased in the H19-TDSC group at the 8week point. C,D) mRNA expression levels of type I and II collagen were detected by RT-qPCR. ${ }^{*} \mathrm{p}<0.05,{ }^{* *} \mathrm{p}<0.01$, relative to control or indicated TDSC group.

human Achilles tendon, leaving their functions to be further explored. ${ }^{41}$ Also, several lncRNAs have been identified from analysis of transcriptomic data from tissue-engineered tendons. ${ }^{42}$ LncRNA KCNQ1OT1 regulates adipogenic and osteogenic differentiation of TDSCs by suppressing miR-138-regulated expression of PPARg and RUNX2. ${ }^{17}$ LncRNA TUG1 regulates osteogenic differentiation of TSPCs by promoting bFGF ubiquitination. ${ }^{43}$ Furthermore, lncRNA H19 accelerates tenogenic differentiation of TDSCs and promotes tendon healing via modulation of miR-29b-3p-regulated TGF-b1 expression. ${ }^{18}$ Further, prior studies suggest that $\mathrm{H} 19$ has potential roles in regulating mammalian muscle growth and development. ${ }^{44}$ In the present study, we found that $\mathrm{H} 19$ was upregulated in RCT samples, and that H19 overexpression in TDSCs promoted tendon healing in RCT by modulating the miR-140-5p/VEGFA axis. In general, H19 is an important regulator for the functional recovery of tendon injuries. However, a broad spectrum of H19-regulated downstream effectors should be further evaluated by RNA-seq or other methods, which will be the topic of future studies.

In summary, we identified a regulatory axis in which H19 targets miR-140-5p to modulate VEGFA expression during tenogenic differentiation of TDSCs in RCT healing. Future studies of the H19/miR-140-5p/VEGFA axis could open a new direction for the development of novel therapies for the clinical treatment of RCT.

\section{References}

1. Chakravarty K, Webley M. Shoulder joint movement and its relationship to disability in the elderly. $\mathrm{J}$ Rheumatol 1993;20:1359-61.

2. Minagawa H, Yamamoto N, Abe H, Fukuda M, Seki N, Kikuchi $\mathrm{K}$, et al. Prevalence of symptomatic and asymptomatic rotator cuff tears in the general population: From mass-screening in one village. J Orthop 2013;10:8-12. 
3. Abate M, Schiavone C, Di Carlo L, Salini V. Prevalence of and risk factors for asymptomatic rotator cuff tears in postmenopausal women. Menopause 2014;21:275-80.

4. Mather RC 3rd, Koenig L, Acevedo D, Dall TM, Gallo P, Romeo A, et al. The societal and economic value of rotator cuff repair. J Bone Joint Surg Am 2013;95:1993-2000.

5. Longo UG, Salvatore G, Rizzello G, Berton A, Ciuffreda M, Candela V, et al. The burden of rotator cuff surgery in Italy: a nationwide registry study. Arch Orthop Trauma Surg 2017; 137:217-24.

6. Boileau P, Brassart N, Watkinson DJ, Carles M, Hatzidakis AM, Krishnan SG. Arthroscopic repair of full-thickness tears of the supraspinatus: does the tendon really heal? J Bone Joint Surg Am 2005;87:1229-40

7. Giombini A, Di Cesare A, Safran MR, Ciatti R, Maffulli N. Shortterm effectiveness of hyperthermia for supraspinatus tendinopathy in athletes: a short-term randomized controlled study. Am J Sports Med 2006;34:1247-53.

8. Bi Y, Ehirchiou D, Kilts TM, Inkson CA, Embree MC, Sonoyama $\mathrm{W}$, et al. Identification of tendon stem/progenitor cells and the role of the extracellular matrix in their niche. Nat Med 2007;13:1219-27.

9. Zhang J, Wang JH. Characterization of differential properties of rabbit tendon stem cells and tenocytes. BMC Musculoskelet Disord 2010;11:10.

10. Salingcarnboriboon R, Yoshitake H, Tsuji K, Obinata M, Amagasa T, Nifuji A, et al. Establishment of tendon-derived cell lines exhibiting pluripotent mesenchymal stem cell-like property. Exp Cell Res 2003;287:289-300.

11. Lui PP. Identity of tendon stem cells — how much do we know? J Cell Mol Med 2013;17:55-64.

12. Lui PP, Chan KM. Tendon-derived stem cells (TDSCs): from basic science to potential roles in tendon pathology and tissue engineering applications. Stem Cell Rev Rep 2011;7:883-97.

13. Cai X, Cai M, Lou L. Identification of differentially expressed genes and small molecule drugs for the treatment of tendinopathy using microarray analysis. Mol Med Rep 2015;11:3047-54.

14. Plachel F, Heuberer P, Gehwolf R, Frank J, Tempfer H, Lehner C, et al. MicroRNA Profiling Reveals Distinct Signatures in Degenerative Rotator Cuff Pathologies. J Orthop Res 2020;38:202-11

15. Chen L, Liu J, Tao X, Wang G, Wang Q, Liu X. The role of Pin1 protein in aging of human tendon stem/progenitor cells. Biochem Biophys Res Commun 2015;464:487-92.

16. Zheng W, Chen C, Chen S, Fan C, Ruan H. Integrated analysis of long non-coding RNAs and mRNAs associated with peritendinous fibrosis. J Adv Res 2019;15:49-58.

17. Yu Y, Chen Y, Zhang X, Lu X, Hong J, Guo X, et al. Knockdown of lncRNA KCNQ1OT1 suppresses the adipogenic and osteogenic differentiation of tendon stem cell via downregulating miR-138 target genes PPARgamma and RUNX2. Cell Cycle 2018;17:2374-85.

18. Lu YF, Liu Y, Fu WM, Xu J, Wang B, Sun YX, et al. Long noncoding RNA H19 accelerates tenogenic differentiation and promotes tendon healing through targeting miR-29b-3p and activating TGF-beta1 signaling. FASEB J 2017;31:954-64.

19. Wang L, Gao W, Xiong K, Hu K, Liu X, He H. VEGF and BFGF expression and histological characteristics of the bone-tendon junction during acute injury healing. J Sports Sci Med 2014; 13:15-21.

20. Asai S, Otsuru S, Candela ME, Cantley L, Uchibe K, Hofmann $\mathrm{TJ}$, et al. Tendon progenitor cells in injured tendons have strong chondrogenic potential: the CD105-negative subpopulation induces chondrogenic degeneration. Stem Cells 2014;32:3266-77.

21. Han CL, Ge M, Liu YP, Zhao XM, Wang KL, Chen N, et al. Long non-coding RNA H19 contributes to apoptosis of hippocampal neurons by inhibiting let-7b in a rat model of temporal lobe epilepsy. Cell Death Dis 2018;9:617.

22. Shin MJ, Shim IK, Kim DM, Choi JH, Lee YN, Jeon IH, et al. Engineered cell sheets for the effective delivery of adiposederived stem cells for tendon-to-bone healing. Am J Sports Med 2020;48:3347-58.

23. Pryce BA, Watson SS, Murchison ND, Staverosky JA, Dunker N, Schweitzer R. Recruitment and maintenance of tendon progenitors by TGFbeta signaling are essential for tendon formation. Development 2009;136:1351-61.

24. Yin Z, Guo J, Wu TY, Chen X, Xu LL, Lin SE, et al. Stepwise differentiation of mesenchymal stem cells augments tendon-like tissue formation and defect repair in vivo. Stem Cells Transl Med 2016;5:1106-16.

25. Li JH, Liu S, Zhou H, Qu LH, Yang JH. starBase v2.0: decoding miRNA-ceRNA, miRNA-ncRNA and protein-RNA interaction networks from large-scale CLIP-Seq data. Nucleic Acids Res 2014;42:D92-7.

26. Agarwal V, Bell GW, Nam JW, Bartel DP. Predicting effective microRNA target sites in mammalian mRNAs. Elife 2015;4.

27. Yoshikawa T, Tohyama H, Enomoto H, Matsumoto H, Toyama Y, Yasuda K. Expression of vascular endothelial growth factor and angiogenesis in patellar tendon grafts in the early phase after anterior cruciate ligament reconstruction. Knee Surg Sports Traumatol Arthrosc 2006;14:804-10.

28. Boyer MI, Watson JT, Lou J, Manske PR, Gelberman RH, Cai SR. Quantitative variation in vascular endothelial growth factor mRNA expression during early flexor tendon healing: an investigation in a canine model. J Orthop Res 2001;19:869-72.

29. Xu Q, Sun WX, Zhang ZF. High expression of VEGFA in MSCs promotes tendon-bone healing of rotator cuff tear via microRNA205-5p. Eur Rev Med Pharmacol Sci 2019;23:4081-8.

30. Zhou YL, Yang QQ, Yan YY, Zhang L, Wang QH, Ju F, et al. Gene-loaded nanoparticle-coated sutures provide effective gene delivery to enhance tendon healing. Mol Ther 2019;27:1534-46.

31. Xing SG, Zhou YL, Yang QQ, Ju F, Zhang L, Tang JB. Effects of nanoparticle-mediated growth factor gene transfer to the injured microenvironment on the tendon-to-bone healing strength. Biomater Sci 2020;8:6611-24.

32. Lahteenmaki HE, Virolainen P, Hiltunen A, Heikkila J, Nelimarkka OI. Results of early operative treatment of rotator cuff tears with acute symptoms. J Shoulder Elbow Surg 2006;15:148-53.

33. Qian L, Yu S, Chen Z, Meng Z, Huang S, Wang P. The emerging role of circRNAs and their clinical significance in human cancers. Biochim Biophys Acta Rev Cancer 2018;1870:247-60.

34. Dubin JA, Greenberg DR, Iglinski-Benjamin KC, Abrams GD. Effect of micro-RNA on tenocytes and tendon-related gene expression: A systematic review. J Orthop Res 2018;36:2823-9.

35. Zhu C, Chen T, Liu B. Inhibitory effects of miR-25 targeting HMGB1 on macrophage secretion of inflammatory cytokines in sepsis. Oncol Lett 2018;16:5027-33.

36. Best KT, Lee FK, Knapp E, Awad HA, Loiselle AE. Deletion of NFKB1 enhances canonical NF-kappaB signaling and increases macrophage and myofibroblast content during tendon healing. Sci Rep 2019;9:10926.

37. Abraham AC, Shah SA, Golman M, Song L, Li X, Kurtaliaj I, et al. Targeting the NF-kappaB signaling pathway in chronic tendon disease. Sci Transl Med 2019;11.

38. Takata A, Otsuka M, Kojima K, Yoshikawa T, Kishikawa T, Yoshida H, et al. MicroRNA-22 and microRNA-140 suppress NF-kappaB activity by regulating the expression of NF-kappaB coactivators. Biochem Biophys Res Commun 2011;411:826-31.

39. Ge Z, Tang H, Lyu J, Zhou B, Yang M, Tang K, et al. Conjoint 
analysis of lncRNA and mRNA expression in rotator cuff tendinopathy. Ann Transl Med 2020;8:335.

40. Ilaltdinov AW, Gong Y, Leong DJ, Gruson KI, Zheng D, Fung DT, et al. Advances in the development of gene therapy, noncoding RNA, and exosome-based treatments for tendinopathy. Ann N Y Acad Sci 2020.

41. Peffers MJ, Fang Y, Cheung K, Wei TK, Clegg PD, Birch HL. Transcriptome analysis of ageing in uninjured human Achilles tendon. Arthritis Res Ther 2015;17:33.

42. Pease LI, Clegg PD, Proctor CJ, Shanley DJ, Cockell SJ, Peffers
MJ. Cross platform analysis of transcriptomic data identifies ageing has distinct and opposite effects on tendon in males and females. Sci Rep 2017;7:14443.

43. Yu Y, Chen Y, Zheng YJ, Weng QH, Zhu SP, Zhou DS. LncRNA TUG1 promoted osteogenic differentiation through promoting bFGF ubiquitination. In Vitro Cell Dev Biol Anim 2020;56:428.

44. Qin CY, Cai H, Qing HR, Li L, Zhang HP. Recent advances on the role of long non-coding RNA H19 in regulating mammalian muscle growth and development. Yi Chuan 2017;39:1150-7.

Received for publication: 28 June 2021. Accepted for publication: 24 July 2021.

This work is licensed under a Creative Commons Attribution-NonCommercial 4.0 International License (CC BY-NC 4.0).

(C) Copyright: the Author(s), 2021

Licensee PAGEPress, Italy

European Journal of Histochemistry 2021; 65:3297

doi:10.4081/ejh.2021.3297 